\title{
MAPAS CONCEITUAIS: UMA POSSIBILIDADE PARA O ESTUDO DOS MODELOS ATÔMICOS EM AULAS DE QUÍMICA
}

\author{
Conceptual maps: a possibility for the study of atomic models in Chemical lessons \\ Mapas conceptuales: una posibilidad para el estudio de los modelos atómicos en \\ clases de Química
}

\author{
Léia Valéria Bocato ${ }^{1}$ \\ Mônica Palacio ${ }^{2}$ \\ Matheus Marques Dias ${ }^{3}$ \\ Wallace Alves $\mathrm{Cabral}^{4}$
}

\begin{abstract}
Resumo
O estudo dos modelos atômicos em aulas de química é fundante para a compreensão de fenômenos mais complexos. Entretanto, conforme pode ser visto nas pesquisas da área, os estudantes tendem a memorizar os conceitos, o que dificulta a relação com outros conhecimentos. Diante disso, esta pesquisa tem por objetivo investigar as potencialidades e as relações intertextuais estabelecidas a partir do estudo de modelos atômicos em uma turma de primeiro ano do Ensino Médio em uma escola pública da cidade de Dourados-MS. A partir de uma sequência de aulas envolvendo experimentação, leitura de um texto de divulgação científica e produção textual, foi possível repensar as práticas que comumente são realizadas. $\mathrm{O}$ foco da análise se deu em torno do mapa conceitual produzido ao final da última aula, percebendo as relações intertextuais estabelecidas e as potencialidades dessas atividades. Os resultados mostraram que, de modo geral, os estudantes compreenderam a dinâmica dos mapas conceituais e conseguiram organizar o conteúdo de modelos atômicos a partir desta ferramenta, possibilitando diferentes relações intertextuais. Além disso, foi possível avaliar os estudantes para além das provas e testes comumente realizadas no ambiente escolar.
\end{abstract}

PALAVRAS-CHAVE: Escrita. Mapas conceituais. Educação Básica. Química.

\section{Abstract}

${ }^{1}$ Licencianda em Química pela Universidade Federal da Grande Dourados-UFGD. Bolsista PIBID/UFGD na Faculdade de Ciências Exatas e Tecnologia. Endereço: Rod. Dourados - Itahum, Km 12 - Cidade Universitária, Dourados/MS. CEP: 79804-970. E-mail: leiabocato@gmail.com

${ }^{2}$ Licencianda em Química pela Universidade Federal da Grande Dourados Bolsista PIBID/UFGD na Faculdade de Ciências Exatas e Tecnologia. Endereço: Rod.Dourados - Itahum, Km 12 - Cidade Universitária, Dourados/MS. CEP:79804-970. E-mail: monicajackeline.p45@gmail.com

${ }^{3}$ Licenciado em Química pela Universidade Estadual de Mato Grosso do Sul-UEMS. Professor de Educação Básica - Química na Escola Estadual Menodora Fialho de Figueiredo. Endereço: Av. Deputado Weimar Gonçalves Torres, 3447 - Jardim Caramuru, Dourados/MS. CEP:79830-020. Telefone: (67) 3424-3158. E-mail: matheusmarquesdias_@hotmail.com

${ }^{4}$ Professor Assistente na Faculdade de Ciências Exatas e Tecnologia da Universidade Federal da Grande Dourados. Doutorando e Mestre em Educação pelo Programa de Pós-Graduação em Educação (PPGE) da Universidade Federal de Juiz de Fora-MG. Endereço: Rod. Dourados - Itahum, Km 12 - Cidade Universitária, Dourados/MS. CEP: 79804-970. E-mail: wallaceacabral@ufgd.edu.br 
The study of atomic models in chemistry lessons is fundamental for the understanding of more complex phenomena. However, as can be seen in the field research, students tend to memorize concepts, which makes it difficult to relate to other knowledge. The purpose of this research is to investigate the potential and intertextual relations established from the study of atomic models in a first-year high school class in a public school in the city of Dourados-MS. From a sequence of classes involving experimentation, reading of a text of scientific divulgation and textual production, it was possible to rethink the practices that are commonly performed. The focus of the analysis was on the conceptual map produced at the end of the last class, perceiving the established intertextual relations and the potentialities of these activities. The results showed that, in general, the students understood the dynamics of the conceptual maps and managed to organize the content of atomic models from this tool, allowing different intertextual relations. In addition, it was possible to evaluate students beyond the tests commonly performed in the school environment.

\section{KEYWORDS: Writing. Concept maps. Basic Education. Chemistry}

\section{Resumen}

El estudio de los modelos atómicos en las clases de química es fundante para la comprensión de fenómenos más complejos. Sin embargo, como se puede ver en las encuestas del área, los estudiantes tienden a memorizar los conceptos, lo que dificulta la relación con otros conocimientos. En este sentido, esta investigación tiene por objetivo investigar las potencialidades y las relaciones intertextuales establecidas a partir del estudio de modelos atómicos en una clase de primer año de la Enseñanza Media en una escuela pública de la ciudad de Dourados-MS. A partir de una secuencia de clases involucrando experimentación, lectura de un texto de divulgación científica y producción textual, fue posible repensar las prácticas que comúnmente se realizan. El foco del análisis se dio en torno al mapa conceptual producido al final de la última clase, percibiendo las relaciones intertextuales establecidas y las potencialidades de esas actividades. Los resultados mostraron que, en general, los estudiantes comprendieron la dinámica de los mapas conceptuales y lograron organizar el contenido de modelos atómicos a partir de esta herramienta, posibilitando diferentes relaciones intertextuales. Además, fue posible evaluar a los estudiantes más allá de las pruebas comúnmente realizadas en el ambiente escolar.

PALABRAS CLAVE: Escrita. Mapas conceptuales. Educación Básica. Química

\section{INTRODUÇÃO}

Sabemos que os conceitos de modelos atômicos normalmente são ensinados nos primeiros anos do Ensino Médio, uma vez que são de fundamental importância para o aprofundamento de questões mais complexas. Devido a sua relevância, alguns autores descrevem possibilidades de atividades e dificuldades em torno do assunto. Por exemplo, Azevedo (2004) destaca que o ensino dos modelos atômicos, de certa forma, tornou-se um assunto que muitos alunos não assimilam muito bem, não gostam e acabam por memorizar, visto que muitas vezes não conseguem estabelecer relações com os outros conceitos da química e para outros contextos do cotidiano.

Indo ao encontro da pesquisa anterior, Melo e Neto (2013) apontam as dificuldades no ensino e aprendizagem dos modelos atômicos. Para eles, um dos problemas está na maneira como o conhecimento é ensinado, na maioria das vezes, os 
estudantes não conseguem relacionar o contexto histórico aos modelos elaborados pelos cientistas da época.

Diante dessas dificuldades, Silva, Machado e Silveira (2015) utilizam os vídeos disponíveis na internet como ferramenta mediadora do processo de ensino e aprendizagem. A partir de uma sequência didática construída, foi possível discutir as limitações de cada modelo e justificar a necessidade histórica de substituí-los ou modifica-los, de maneira a possibilitar as explicações científicas dos diversos fenômenos observados.

No artigo de Medeiros, Medeiros e Ramalho Neto (2013), “A construção dos modelos atômicos em uma abordagem histórica à luz da natureza do conhecimento científico" (p. 1) propiciou momentos importantes para a reformulação do método de ensinar sobre os modelos atômicos. Conforme pode ser visto nessa pesquisa, a utilização da dinâmica da caixa favoreceu a ideia de um modelo consensual pelos alunos. Além disso, houve discussões para conduzir os estudantes a questionarem sobre os modelos elaborados pelos mesmos. Por fim, "os resultados indicam que houve uma compreensão da proposta pelos alunos e foi verificada uma grande dificuldade na verbalização das leis ponderais e estrutura atômica" (p. 3)

Além das dificuldades e possibilidades a partir do trabalho com modelos atômicos, outra resistência encontrada em aulas de Química é o não incentivo da escrita. Cassiani e Almeida (2005) destacam que muitos registros escritos produzidos pelos estudantes tendem a apresentar um apego à memorização mecânica e à repetição empírica, na tentativa de repetir fielmente o que foi discutido em sala de aula. Seus estudos mostram um deslocamento nessa tendência após o trabalho com diferentes formas de escrita nas aulas de ciências. Dentro disso, Orlandi (2012) apresenta três tipos de repetição que podem aparecer nas produções escritas:

a) A repetição empírica (mnemônica), que é a do efeito papagaio, só repete;

b) A repetição formal (técnica), que é um outro modo de dizer o mesmo;

c) A repetição histórica, que é a que desloca, a que permite o movimento, porque historiciza o dizer do sujeito [...]. (p. 54).

É comum nas atividades dos alunos da Educação Básica o uso constante da repetição empírica, havendo, muitas vezes, cópias dos livros didáticos ou o uso de exercícios com respostas diretas. Consideramos esse tipo de repetição frequente em diferentes níveis de escolarização, conforme aponta Flôr (2009). Esse tipo de escrita é muito difundida pelos estudantes: primeiro eles leem as questões propostas para o texto, depois localizam nele as palavras-chave referentes a essas questões e por fim transcrevem essa parte do texto para a resposta.

Dentre as diferentes possibilidades de valorização da escrita em aulas de Química, optamos, nessa pesquisa, pelo incentivo à construção de mapas conceituais, buscando diferentes movimentos de autoria para além da repetição empírica. Em geral, os mapas conceituais são diagramas, esquematizações ou representações gráficas, construídos através de palavras chave que se interligam, facilitando a compreensão e a interpretação de conceitos de um determinado conteúdo.

Por se tratar de uma ferramenta de aprendizagem, os mapas conceituais podem ser construídos de diferentes formas, dependendo do indivíduo, ou seja, são individuais e intrasferíveis. Tal recurso deve ser construído a partir de um tema central e sendo interligado de forma prática a conceitos secundários. Para auxiliar na construção, 
utilizam-se setas, figuras geométricas e palavras de ligação. Essas características não são obrigatórias; o importante ao traçar um mapa é a capacidade em evidenciar significados atribuídos a conceitos e saber relacionar esses para a construção do conhecimento de um determinado conteúdo.

Em geral, os mapas conceituais podem ser usados para mostrar relações significativas entre conceitos ensinados em uma única aula, em uma unidade de estudo ou em um curso inteiro. São representações concisas das estruturas conceituais que estão sendo ensinadas e, como tal, provavelmente facilitam a aprendizagem dessas estruturas. Entretanto, diferentemente de outros materiais didáticos, os mapas não são autoinstrutivos: devem ser explicados pelo professor. Além disso, embora possam ser usados para dar uma visão geral do tema em estudo, é importante usá-los quando os alunos já têm uma certa familiaridade com o conteúdo trabalhado, de modo que sejam potencialmente significativos e permitam a integração, reconciliação e diferenciação de significados de conceitos (MOREIRA, 1980). Para esse trabalho, acreditamos nas potencialidades dos mapas conceituais como um recurso que possibilita a articulação do conhecimento, incentivo à escrita e no auxílio ao docente no processo avaliativo.

Segundo o autor anteriormente citado, algumas vantagens e desvantagens são apresentadas no ensino com o uso dos mapas conceituais pelo professor.

Entre as possíveis vantagens, pode-se mencionar:

1. Enfatizar a estrutura conceitual de uma disciplina e o papel dos sistemas conceituais em seu desenvolvimento;

2. Mostrar que os conceitos de uma certa disciplina diferem quanto ao grau de generalidade, apresentando esses conceitos em uma ordem hierárquica de que facilite sua aprendizagem;

Dentre as possíveis desvantagens, podemos citar:

1. Se o mapa não tem significado para os alunos, eles podem encara-lo como algo mais a ser memorizado;

2. Os mapas podem ser muito complexos ou confusos e dificultar a aprendizagem, ao invés de facilitá-las, caso não seja trabalhado de maneira adequada;

A partir do momento em que os alunos utilizam os mapas conceituais para integrar, relacionar e diferenciar conceitos, analisar textos, capítulos de livros e até mesmo experimentos laboratoriais, eles estarão usando essa ferramenta como recurso de aprendizagem. Ou seja, mediante o contato com os mapas conceituais como ferramenta para o recurso da aprendizagem é possível ter uma visão geral da organização conceitual que o aluno adquiriu de um determinado conteúdo por meio de um mapeamento avaliativo qualitativo. Nesse contexto, acreditamos nos mapas conceituais como possibilidade de estabelecer diferentes relações intertextuais, que, para Orlandi (2012), remete ao fato de que um texto nasce em outros textos, assim como, também, aponta para outros tantos.

Diante dessas discussões, essa pesquisa tem como objetivo geral investigar as potencialidades e as relações intertextuais estabelecidas a partir do estudo de modelos atômicos em uma turma de primeiro ano do Ensino Médio em uma escola pública da cidade de Dourados no Estado do Mato Grosso do Sul. 


\section{Caminhos metodológicos}

As atividades aqui descritas fazem parte das ações previstas no âmbito do Programa Institucional de Bolsas de Iniciação à Docência (PIBID) do curso de licenciatura em Química da Universidade Federal da Grande Dourados (UFGD). Tais ações foram planejadas por duas bolsistas do projeto e orientadas pelo supervisor da escola e orientador pertencente à universidade.

Durante quatro meses, houve o estudo e planejamento das atividades pelas bolsistas, bem como o reconhecimento do ambiente escolar. Após esse processo, no $2^{\circ}$ bimestre escolar de uma escola pública de Dourados, quatro turmas de primeiro ano vivenciaram as atividades descritas no quadro 1 . Devido a quantidade de material produzido, optamos pela análise dos mapas conceituais de apenas uma turma. Selecionamos a única classe em que a atividade avaliativa foi desenvolvida no ambiente escolar, as demais, por conta da limitação do tempo, realizaram a atividade em casa para entrega no próximo encontro.

\begin{tabular}{|c|c|}
\hline \multicolumn{2}{l}{ Quadro 1: Síntese das atividades desenvolvidas. } \\
\hline AULAS & ATIVIDADES DESENVOLVIDAS \\
\hline $\mathbf{1}$ & $\begin{array}{c}\text { - Resolução de uma lista de exercícios com os estudantes; } \\
\text { - Atividade experimental: teste de chama; }\end{array}$ \\
\hline $\mathbf{2}$ & $\begin{array}{c}\text { - Conhecendo os mapas conceituais: principais conceitos; } \\
\text { - Leitura do texto "A mágica dos fogos de artifícios" e produção de um } \\
\text { mapa conceitual a partir dele; }\end{array}$ \\
\hline $\mathbf{3}$ & $\begin{array}{c}\text { - Atividade avaliativa: construção de um mapa conceitual referente ao } \\
\text { conteúdo de modelos atômicos. }\end{array}$ \\
\hline
\end{tabular}

Fonte: elaborado pelos autores.

Com intuito de aprofundar na discussão das atividades apresentadas no quadro 1, abaixo, faço uma breve apresentação das ações desenvolvidas aula a aula.

Aula 1: Inicialmente foi entregue uma lista de exercícios com intuito de verificar o entendimento dos alunos sobre o conteúdo já trabalhado. Nesse processo, as duas bolsistas foram mesa a mesa dialogando com os estudantes e sanando as possíveis dúvidas. Após essa atividade, uma experimentação investigativa foi realizada, a partir do teste de chamas, hipóteses foram elucidadas buscando explicações para tais fenômenos.

Aula 2: A partir da criação de um conjunto de slides, as bolsistas apresentaram os principais aspectos que devem conter um mapa conceitual. Para facilitar a compreensão dos alunos, foi sugerida a leitura do texto "A mágica dos fogos de artifícios"5 e a criação de um mapa conceitual a partir dele. Esse primeiro momento de produção textual permitiu aos discentes compreender a dinâmica de construção desse gênero e a importância dele para sintetizar informações.

Aula 3: Como atividade avaliativa prevista para o segundo bimestre, os estudantes tiveram que produzir um mapa conceitual a partir do conteúdo de modelos atômicos.

Visando resguardar a identidade dos sujeitos envolvidos, os estudantes serão identificados por nomes fictícios criados pelos autores.

${ }^{5} \mathrm{O}$ texto está disponível no link: https://www.ufmg.br/cienciaparatodos/wp-content/uploads/2012/03/03amagicadosfogosdeartificio.pdf 


\section{Mapas conceituais: possibilidades e desafios a partir das atividades dos estudantes}

A partir da atividade avaliativa produzida ao final da terceira aula em torno do conteúdo de modelos atômicos, serão analisadas, neste tópico, as potencialidades e as limitações dos mapas conceituais dos discentes. Como pode ser visto no mapa conceitual produzido pela aluna Bruna (imagem 1), há uma centralidade com relação ao tema (modelos atômicos) e a ligação com quatro cientistas que propuseram a explicação para o átomo. Além disso, as figuras desenhadas demonstram a aproximação com os modelos elaborados pelos cientistas, tal como é representado no livro didático de Química adotado pela escola.

É perceptível também o entendimento do conteúdo pela estudante ao apresentar corretamente os principais conceitos aos respectivos cientistas, fazendo uso de setas, palavras chave e figuras, facilitando a compreensão do leitor. Entretanto, na organização desse mapa conceitual, não é possível identificar os aspectos históricos e quais propostas vieram primeiro, o que contrapõe o defendido por Melo e Neto (2013).

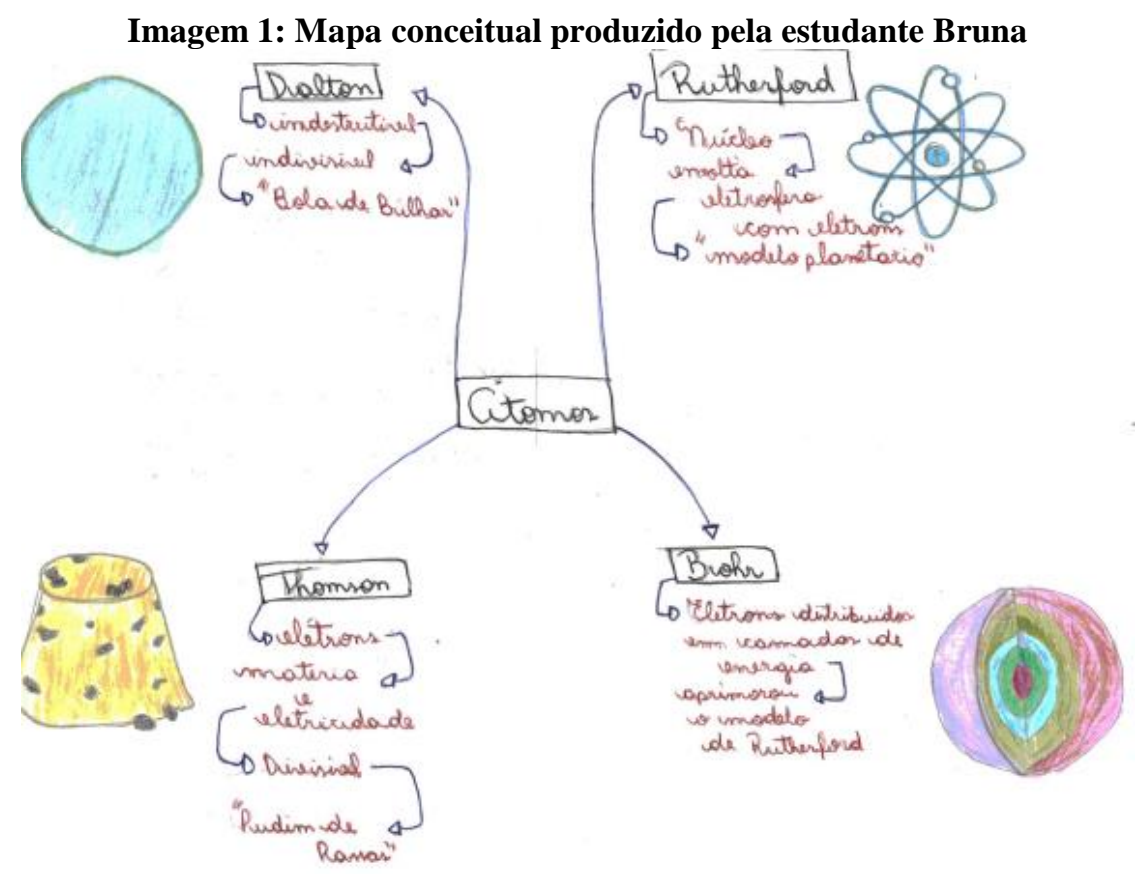

Fonte: Elaborado pela estudante Bruna.

Tal como Bruna, o estudante Pedro também produziu o mapa conceitual a partir dos pressupostos teóricos indicados na aula 2. Os quatro cientistas descriminados nas aulas como fundamentais para o entendimento de fenômenos mais complexos foram apresentados em seu teto. Diferentemente da estudante anteriormente citada, a organização dos pesquisadores de maneira linear, possibilita a compreensão cronológica da evolução atômica. Porém, podemos dizer que o mapa conceitual, ao tentar generalizar conteúdos, dificulta as relações entre eles, podendo apresentar para um leitor leigo a linearidade na construção do conhecimento científico.

Apesar da atividade indicada ser igual para todos os alunos (produza um mapa conceitual a partir do conteúdo modelos atômicos) é interessante perceber as 
diferentes organizações dos mapas e os níveis de aprofundamento. Para o estudante Pedro, outras palavras chave foram apresentadas, por exemplo, os termos prótons, nêutrons, elétrons e outros. Essas diferentes produções a partir do mesmo conteúdo evidenciam a não transparência da linguagem, apontando que cada sujeito carrega suas histórias de leituras e que estas influenciarão nas suas decisões (ORLANDI, 2012).

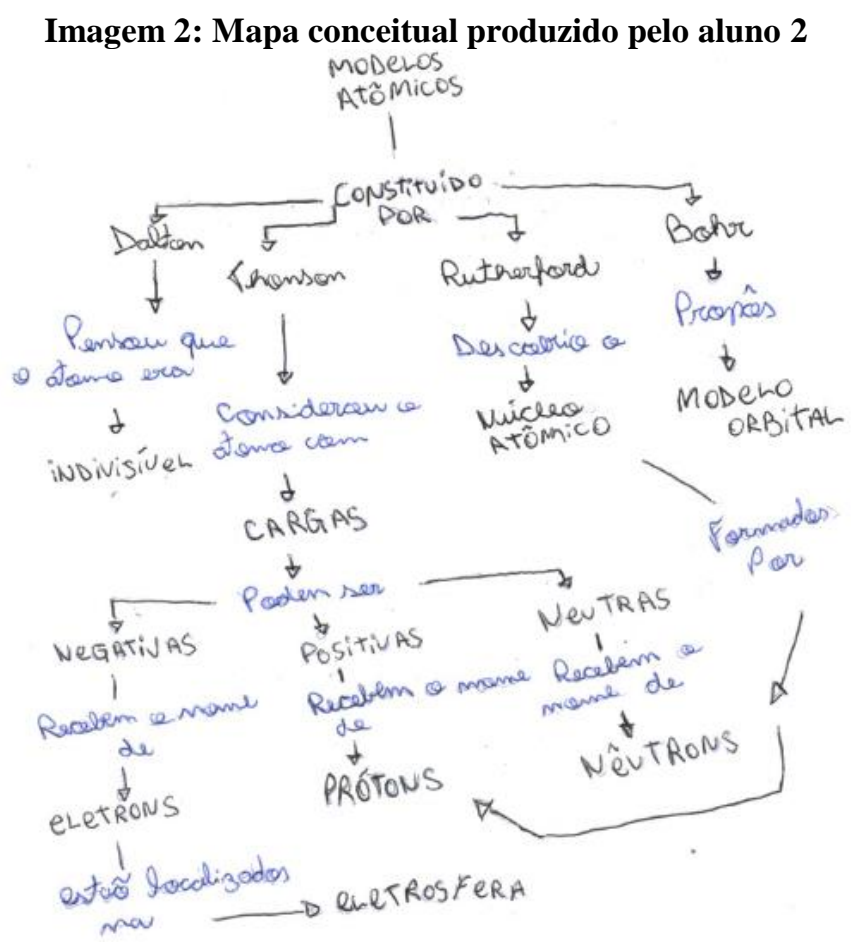

Fonte: Produzido pelo estudante Pedro.

Assim como os estudantes anteriormente citados, a discente Maria compreendeu os princípios que balizam um mapa conceitual, fazendo uso de palavras chave, setas e a tentativa de englobar os diferentes conceitos estudados. Contudo, há uma confusão teórica em alguns momentos, por exemplo, ao associar o termo "eletrosfera" ao cientista Thomson e ao confundir Isaac Newton com a proposta de Dalton. Esses equívocos podem sinalizar para o docente a necessidade de retomada dos principais conceitos e a sinalização para o aluno dos erros. Sendo assim, acreditamos nas potencialidades dos mapas conceituais também como uma atividade avaliativa, ampliando os métodos avaliativos para além dos testes e provas. 
Imagem 3: Mapa conceitual produzido pela aluna 3

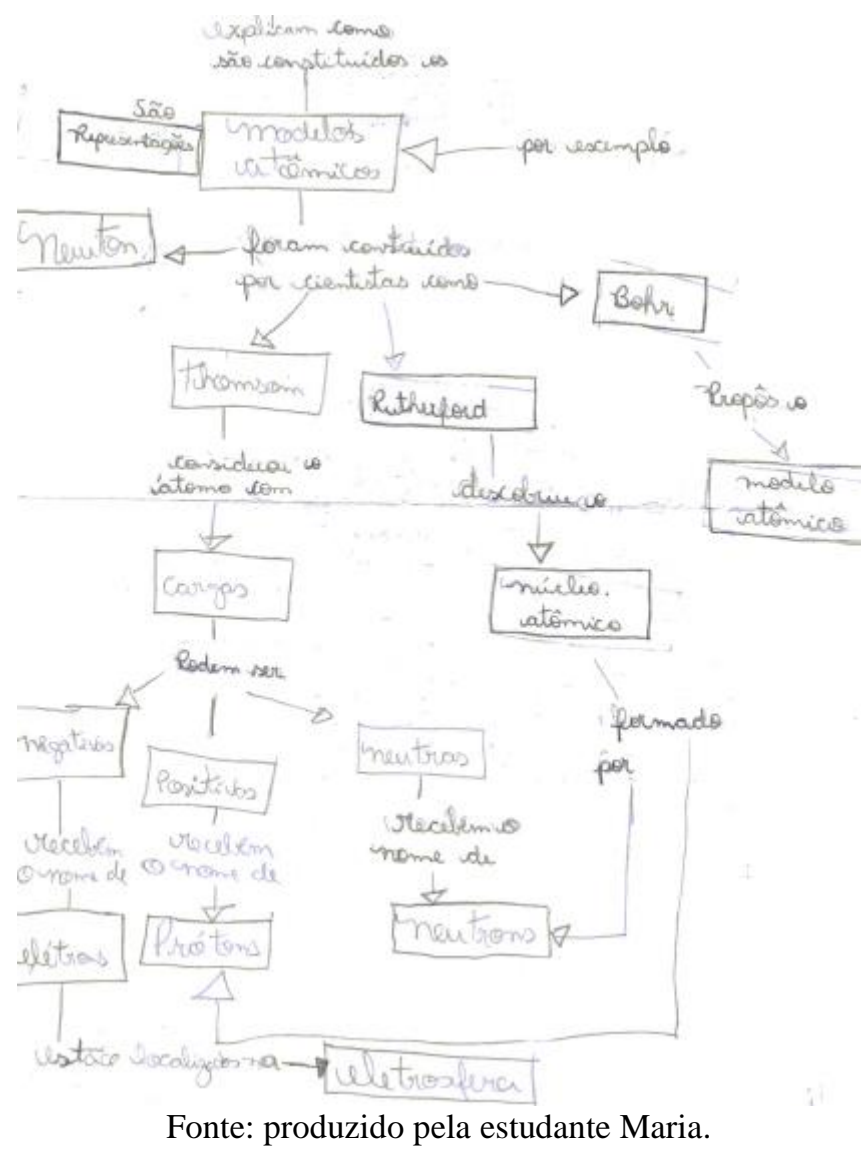

Apesar das especificidades em cada produção, esses três mapas conceituais são representativos para a turma em questão. Na tentativa de fazer uma síntese das 15 atividades produzidas pelos estudantes, estabelecemos quatro critérios, sendo eles:

$1^{\text {o }}$ critério: Presença do tema central (modelos atômicos), utilização de setas, palavras chave e figuras (opcional), correta utilização dos conceitos e teóricos.

$2^{\circ}$ critério: Compreendeu a dinâmica de construção dos mapas conceituais, porém, cometeu erros conceituais. conceitual.

$3^{\text {o }}$ critério: Domínio conceitual do conteúdo e dificuldade de criação de um mapa

$4^{\text {o }}$ critério: não compreendeu o conteúdo e a atividade proposta.

Mediante a construção desses eixos, foram realizadas diversas leituras dos mapas conceituais, aproximando cada proposta de um critério. Oito estudantes se enquadraram no primeiro critério, construindo um mapa conceitual de forma correta sobre o conteúdo trabalhado ( $1^{\circ}$ critério). Dois alunos conseguiram construir o mapa conceitual, porém, cometeram erros teóricos, tal como foi apresentado pela estudante Maria. No terceiro critério, tivemos 3 alunos e, no último, dois alunos. 


\section{CONSIDERAÇÕES FINAIS}

A partir do desenho analítico aqui apresentado, é possível inferir que, de modo geral, os estudantes compreenderam as potencialidades dos mapas conceituais e conseguiram organizar o conteúdo de modelos atômicos a partir desta ferramenta. Além disso, foi possível avaliar os estudantes para além das provas e testes comumente realizadas no ambiente escolar, possibilitando (re)pensar os processos avaliativos, bem como as possibilidades de escrita.

$\mathrm{Na}$ visão das bolsistas pertencentes ao projeto PIBID, tal sequência didática permitiu uma aproximação sobre o pensar sobre a Química com tanta intensidade quanto o fazem sobre o pensar quimicamente. Nesse sentido, os aspectos sociais e históricos na evolução dos mapas conceituais afloraram.

\section{REFERENCIAS}

AZEVEDO, M.C.P.S. Ensino por investigação: problematizando as atividades em sala de aula. In: CARVALHO, A.M.P. Ensino de ciências - unindo a pesquisa e a prática. São Paulo: Pioneira Thomson Learning, p. 19-33, 2004.

CASSIANI, S; ALMEIDA, M. J. P. M. Escrita no ensino de Ciências: autores do ensino fundamental. Ciência \& Educação. Bauru. v.11, n.3, p. 367-382, 2005.

FLÔR, C. C. Leitura e formação de leitores em aulas de química no ensino médio. Tese de Doutorado. PPGECT/UFSC. 2009.

MELO, M. R.; NETO, E. G. L. Dificuldades de Ensino e Aprendizagem dos Modelos Atômicos em Química. Química Nova na Escola, v. 35, n. 2, p. 112-122, 2013.

MOREIRA, M. A. Mapas conceituais como instrumentos para promover a diferenciação conceitual progressiva e a reconciliação integrativa. Ciência e Cultura, 32(4): 474-479, 1980.

ORLANDI, E. P. Análise de Discurso: princípios e procedimentos. 10.ed. Campinas, Pontes Editores, 2012.

SILVA, G. R; MACHADO, A. H; A. S; SILVEIRA, K. P. Modelos para o Átomo: Atividades com a Utilização de Recursos Multimídia. Modelos Para O Átomo, São Paulo-sp, v. 37, n. 2, p.106-111, 2015.

MEDEIROS, L. L; MEDEIROS, G. H; RAMALHO NETO, O. E. A construção dos modelos atômicos em uma abordagem histórica à luz da natureza do conhecimento científico: uma experiência do PIBID Química da UFRN. In: Anais do IX Encontro Nacional de Pesquisa em Educação em Ciências, Águas de Lindóia, 2013. 
ISSN 2237-258X

Recebido em: 14/06/2017

Aprovado em: 10/07/2017 